\title{
miR-148a-3p regulates alcoholic liver fibrosis through targeting ERBB3
}

\author{
JIE XIONG $^{1 *}$, JIANBO NI $^{2^{*}}$, CONGYING CHEN $^{2}$ and KEZHOU WANG ${ }^{3}$ \\ ${ }^{1}$ Department of Gastroenterology and Hepatology, Tongji Hospital, Tongji University School of Medicine, \\ Shanghai 200065; ${ }^{2}$ Department of Gastroenterology and Hepatology, Shanghai General Hospital, \\ Shanghai Jiao Tong University School of Medicine, Shanghai 200080; ${ }^{3}$ Department of Pathology, \\ Xinhua Hospital Affiliated to Shanghai Jiao Tong University School of Medicine, Shanghai 200092, P.R. China
}

Received July 29, 2019; Accepted April 14, 2020

DOI: $10.3892 /$ ijmm.2020.4655

\begin{abstract}
Alcoholic liver disease greatly affects human health. Previous studies have identified that microRNAs (miRNAs) are associated with the pathogenesis of alcoholic liver fibrosis (ALF). Therefore, the present study explored the regulatory mechanism of miR-148a-3p in ALF. An ALF model was established in rats by alcohol gavage, followed by treatment with miR-148a-3p. Reverse transcription-quantitative (RT-q) PCR was performed to detect miR-148a-3p expression in the rat liver tissues. The levels of lactate dehydrogenase (LDH), aspartate aminotransferase (AST), alanine transaminase (ALT) and alkaline phosphatase (ALP) were determined by enzyme-labeled colorimetry. Liver damage was evaluated by liver indices and histology. The direct target gene of miR-148a-3p was predicted by a dual luciferase reporter assay. The effects of miR-148a-3p and miR-148a-3p in combination with receptor tyrosine-protein kinase erbB-3 (ERBB3) on HSC-T6 cell viability and apoptosis were detected by MTT and flow cytometry assays, respectively. Western blotting and RT-qPCR assays were performed to detect the expression levels of proteins and mRNA associated with fibrosis and apoptosis. The data showed that miR-148a-3p mimics inhibited the expression levels of AST, ALT, ALP, LDH, $\alpha$-SMA and type I collagen in the model, decreased the liver indices, and improved the liver damage caused by alcohol. ERBB3, which was predicted as the direct target gene of
\end{abstract}

Correspondence to: Dr Jie Xiong, Department of Gastroenterology and Hepatology, Tongji Hospital, Tongji University School of Medicine, 389 Xincun Road, Putuo, Shanghai 200065, P.R. China E-mail: doctorxiongj@163.com

*Contributed equally

Abbreviations: LDH, lactate dehydrogenase; AST, aspartate aminotransferase; ALT, alanine transaminase; ALP, alkaline phosphatase; miRNA, microRNAs; RT-qPCR, reverse transcriptionquantitative $\mathrm{PCR}$; NC, negative control

Key words: micoRNA-148a-3p, alcoholic liver disease, receptor tyrosine-protein kinase erbB-3, cell viability, apoptosis
miR-148a-3p, reversed the effects of ERBB3 on promoting cell viability and inhibiting apoptosis. Concomitantly, miR-148a-3p reversed the increased expression of Bcl-2 and inhibited the expression levels of Bax and c-cleaved-3 caused by ERBB3. These data suggested that miR-148a-3p regulated ALF and the viability and apoptosis of hepatic stellate cells through targeting ERBB3.

\section{Introduction}

Alcoholic liver disease is a chronic liver disease caused by long-term alcohol intake.Early manifestations of the disease are fatty liver, alcoholic hepatitis, liver fibrosis and liver cirrhosis, which also occurs during the disease progression, causing extensive necrosis of liver cells and even liver failure when the disease is severe $(1,2)$. Increasing levels of alcohol consumption has led to an increase in the incidence of alcoholic liver disease. It has been reported that alcohol-associated liver deaths accounted for up to $48 \%$ of cirrhosis-associated deaths in the United States in 2007 and are also major contributors to liver disease-related mortality in other countries (3). Data showed that up to $90 \%$ of patients with heavy alcohol intake have fatty liver, which could be rapidly reversible with abstinence (4). If the alcohol abuse persists, liver fibrosis progressively develops, ultimately resulting in cirrhosis $(4,5)$. Evidence indicates that multiple pathways are involved in the progression of alcoholic liver disease. For example, ethanol induces binding of lipopolysaccharides to monocyte differentiation antigen CD14, which then combines with Toll-like receptor 4 to activate multiple cytokine genes involved in inflammatory cytokine production and to decrease the expression of STATs in Kupffer cells, thereby causing alcoholic liver disease (6). Besides, long-term alcohol consumption also induces mitochondrial damage, reactive oxygen species production and lipid peroxidation, which cause liver injury $(7,8)$.

Previous reports have revealed that the activation of hepatic stellate cells serves a critical role in hepatic fibrosis development $(9,10)$. Activated hepatic stellate cells exhibit a proliferative and myofibroblastic phenotype with increased production of type I collagen and other extracellular matrix proteins. A recent report showed that suppression of YAP can alleviate the development of hepatic fibrosis through enhancing 
apoptosis and reversion of hepatic stellate cells (11). Another study indicated that silencing of NADPH oxidase 5 can inhibit the proliferation and collagen type I levels in hepatic stellate cells (12). Nevertheless, the factors that regulate the proliferation and apoptosis of hepatic stellate cells have not been comprehensively identified yet.

Previous studies have identified that that microRNAs (miRNAs) are associated with the pathogenesis of alcoholic liver fibrosis (ALF) (13-15). miRNAs measure 20-25 nucleotides in length, and serve important roles in regulating the expression levels of cellular genes and proteins $(16,17)$. It was also revealed that genes from the miR-148 family function critically in the liver by promoting a liver-specific phenotype and inhibiting invasion in transformed cells $(18,19)$. miR-148a-3p has been demonstrated to act as a tumor suppressor by participating in the occurrence and development of various tumors (20-22). An integrated analysis of miRNAs from Gene Expression Omnibus datasets showed that miR-148a-3p is one of a number of functional regulatory modules in the development of liver fibrosis (23). Notably, some studies indicate that miR-148a-3p had a negative effect on hepatic stellate cell activation by targeting transforming growth factor $\beta$ receptor type (TGFBR)1 and TGFBR2 (24) or ubiquitin carboxyl-terminal hydrolase 4 (25). Overexpression of miR-148a-3p also promotes autophagic and apoptotic activity of hepatic stellate cells (26). However, the role and molecular mechanisms of miR-148a-3p in liver fibrosis and activation of hepatic stellate cells remain largely unknown.

Receptor tyrosine-protein kinase erbB-3 (ERBB3) is a membrane bound protein that belongs to the epidermal growth factor receptor (EGFR/ERBB) family of receptor tyrosine kinases. Several studies have indicated that ERBB3 participates in various liver diseases including liver fibrosis (27-29). A recent study indicated that ERBB3 can be regulated by miR-125a-5p in hepatitis B virus-associated hepatocellular carcinoma (30). Whether ERBB3 can be regulated by miR-148a-3p and participated in the viability of hepatic stellate cells requires further exploration.

miRNAs can regulate disease processes by identifying target genes and degrading themselves or inhibiting the expression of target genes according to different degrees of complementarity (31). TargetScan7.2 (32) is a well-known online analysis tool by which the potential interaction between miRNAs and mRNAs can be predicted. Rat hepatic stellate cells HSC-T6 have been commonly used in the study of hepatic stellate cells in $\operatorname{ALF}(33,34)$. In the present study, TargetScan7.2 predicted that ERBB3 was the direct target gene for miR-148a-3p, and based on this, the association between miR-148a-3p and ERBB3 was studied, and their roles in the pathogenesis of alcoholic liver fibrosis were further investigated in HSC-T6 cells.

\section{Materials and methods}

Establishing ALF model in rats. Male Sprague-Dawley rats (8 weeks old; weight, 278-289 g) were purchased from Tongji Hospital, Tongji University School of Medicine. Rats were kept in cages at room temperature $\left(23 \pm 3^{\circ} \mathrm{C}\right)$ with a constant humidity $(50 \pm 10 \%)$ with free access to food/water and a 12-h/12-h light/dark cycle. The animal experimental protocol was approved by the Tongji Hospital of Tongji University and performed in accordance with the Guidance and Ethics Committee of Tongji Hospital, Tongji University School of Medicine (approval no. THTU20160810A). Lentiviral vectors with overexpressed miR-148a-3p and negative controls were synthesized by Sangon Biotech Co., Ltd. The rats were randomly divided into 5 groups: Control; miR-148-3p; Model; Model + NC; and Model + miR-148-3p, with 16 rats in each group. Rats in the miR-148-3p and Model + miR-148-3p groups were selected and injected with $200 \mu 1$ overexpressed miR-148a-3p lentivirus vectors, while the rats in the Model $+\mathrm{NC}$ group were injected with $200 \mu \mathrm{l}$ negative control (NC) vectors. For the surgical procedure, the rats were held in place, and their blood vessels were gently rubbed with an incandescent lamp and repeatedly swabbed by $75 \%$ alcohol (cat. no. 10009218, Sinopharm Chemical Reagent Beijing Co., Ltd.) to fully dilate the vessels and soften the cuticle of the skin to facilitate puncture. The lentivirus and NC were slowly injected into one-third of the tail vein. The control and Model groups were administered distilled water by gavage. The next day, the rats from the model group received irrigation with $56 \%$ alcohol $(9.2 \mathrm{mg} / \mathrm{kg})$ once a day. Rats from the control group received irrigation with double-distilled water $(10 \mathrm{ml} / \mathrm{kg})$ once a day. During the protocol, all rats were fed with standard food and water.

The Model, Model + NC and Model + miR-148-3p groups were used to establish the ALF model. Briefly, the rats were treated by alcohol gavage for 9 weeks. At the end of 9 weeks, all rats were anesthetized by intramuscular injections of $60 \mathrm{mg} / \mathrm{kg}$ ketamine (cat. no. ZTR-K165295; Shanghai ZZBIO Co., Ltd.) and $10 \mathrm{mg} / \mathrm{kg}$ of xylazine (cat. no. B27154, Shanghai Yuanye Bio-Technology Co., Ltd.) mixed in a 1:1 ratio. The blood was then collected via the posterior orbital venous plexus of the rats. All rats were then injected with an overdose of ketamine $(180 \mathrm{mg} / \mathrm{kg})+$ xylazine $(30 \mathrm{mg} / \mathrm{kg})$ immediately for euthanasia. The weight of the animals at the time of sacrifice was at 300.06-319.69 g. Then, the complete livers were removed from the abdominal cavity immediately. The liver tissues were weighed and the liver index was calculated using the following formula: Liver index=wet weight of the liver/body weight. The blood was allowed to coagulate at room temperature for $2 \mathrm{~h}$, and then centrifuged at $1,000 \mathrm{x}$ at $4^{\circ} \mathrm{C}$ for $20 \mathrm{~min}$. The serum was removed and cryopreserved at $-20^{\circ} \mathrm{C}$ for serum detection. Half of the liver was immersed in $10 \%$ neutral formalin (cat. no. 181101; Nanchang Yulu Experimental Equipment Co., Ltd.) for histological analysis, while the other half was placed in liquid nitrogen and maintained at $-80^{\circ} \mathrm{C}$ for western blotting and reverse transcription-quantitative (RT-q)PCR test.

Hematoxylin and eosin $(H \& E)$ staining. The tissues $(1 \times 1 \mathrm{~cm})$ were fixed using $10 \%$ neutral formalin, dehydrated by a graded ethanol series $(80,90,95$ and $100 \%)$ for $2 \mathrm{~h}$ and then treated with $50 \%$ xylene for $30 \mathrm{~min}$. Next, the tissues were immersed in wax, embedded and sectioned. The sections were examined using H\&E staining. The samples were dewaxed by xylene (cat. no. 10023418; Sinopharm Chemical Reagent Co., Ltd.) and then dephenylated using a graded ethanol series (100, 95,80 and $70 \%$ ) for $2 \mathrm{~min}$. Next, the tissues were rehydrated by rinsed in distilled water twice to, stained using $0.5 \%$ hematoxylin (Beijing Zhongshan Jinqiao Biotechnology Co., Ltd.) for $20 \mathrm{~min}$ at room temperature and then washed under 
Table I. Primers used in reverse transcription quantitative PCR analysis.

\begin{tabular}{ll}
\hline Gene & \multicolumn{1}{c}{ Primer sequence } \\
\hline miR-148a-3p & Forward: 5'-AGCAGTTCAGTGCACTACAG-3' \\
R-SMA & Feverse: 5'-GCAGGGTCCGAGGTATTC-3' \\
Collagen I & Reverse: 5'-CCACCGATCCAGACAGACTA-3' \\
Bcl-2 & Forward: 5'-TCACCTACTGCACGCTTGTGG-3' \\
& Reverse: 5'-TTGGCTTTTGGGGAAATTGA-3' \\
Bax & Forward: '5'-TGCACCTGAGCGCCTTCAC-3' \\
& Reverse: 5'-TAGCTGATTCGACCATTTGCCTGA-3' \\
ERBB3 & Forward: '5'-CCACCAGCTCTGAACAGTT-3' \\
& Reverse: 5'-TCAGCCCATCTTCTTCCAG-3' \\
GAPDH & Forward: '5'-AGATCTGCACCATTGACGTC-3' \\
& Reverse: 5'-TAGGTCTAGGTCCAGTTCTG-3' \\
& Forward: 5'-CCAACCGCGAGAAGATGA-3' \\
& Reverse: 5'-CCAGAGGCGTACAGGGATAG-3' \\
\hline
\end{tabular}

miR, microRNA; $\alpha$-SMA, $\alpha$-smooth muscle actin; ERBB3, receptor tyrosine-protein kinase erbB-3.

running water. The slices were then rinsed in acidification solution comprised of hydrochloric acid (cat. no. 10011008; Sinopharm Chemical Reagent Co., Ltd.) and $75 \%$ ethanol at a ratio of 1:99 for $1 \mathrm{~min}$ to remove the blue-stained cytoplasm and then washed with tap water for $10 \mathrm{~min}$. Then the tissues were stained using $0.5 \%$ eosin (Beijing Zhongshan Jinqiao Biotechnology Co., Ltd.) for $15 \mathrm{~min}$ at room temperature, dehydrated in $100 \%$ ethanol for $15 \mathrm{~min}$ and treated with xylene for $15 \mathrm{~min}$. Finally, neutral gum was used to seal the film, and pathological changes were observed using a CKX31 light optical microscope (magnification, x100 and x200; Olympus Corporation).

Treated culture. The rat hepatic stellate HSC-T6 cell line was purchased from Shanghai Institutes for Biological Sciences, Chinese Academy of Sciences. The cells were cultured in RPMI medium 1640 (Gibco; Thermo Fisher Scientific, Inc.) containing $10 \%$ fetal bovine serum (FBS; Gibco; Thermo Fisher Scientific, Inc.) at $37^{\circ} \mathrm{C}$ in a $5 \% \mathrm{CO}_{2}$ atmosphere.

Detection of lactate dehydrogenase ( $\mathrm{LDH})$, aspartate aminotransferase (AST), alanine transaminase (ALT) and alkaline phosphatase (ALP) activity. The activities of LDH, AST, ALT and ALP were detected by LDH (cat. no. MAK066; Sigma-Aldrich; Merck KGaA), AST (cat. no. MAK055; Sigma-Aldrich; Merck KGaA), ALT (cat. no. MAK052; Sigma-Aldrich; Merck KGaA) and ALP (cat. no. MAK262; Sigma-Aldrich; Merck KGaA) assay kits. The cells were inoculated in a 96-well plate and allowed to adhere to the walls of the wells. In each group, blank and standard wells were generated, and double-distilled water (25 $\mu \mathrm{l})$ and matrix buffer $(25 \mu \mathrm{l})$ were added into the blank wells, while double distilled water $(5 \mu \mathrm{l}), 0.2 \mathrm{mmol} / \mathrm{l}$ standard solution (20 $\mu \mathrm{l})$ and matrix buffer $(25 \mu \mathrm{l})$ were added into standard wells. In each group, testing and control wells were generated, and the cell suspension $(20 \mu \mathrm{l})$, matrix buffer $(25 \mu \mathrm{l})$ and coenzyme I (5 $\mu \mathrm{l})$ were added into the testing wells, while the cell suspension $(20 \mu \mathrm{l})$, matrix buffer $(25 \mu \mathrm{l})$ and double distilled water
(5 $\mu \mathrm{l}$ ) were added into the control well. The 96-well plate was held in a water bath at $37^{\circ} \mathrm{C}$ for $15 \mathrm{~min}$, then 2, 4-dinitrobenzene hydrazine $(25 \mu \mathrm{l})$ was added into each group. Next, the cells were mixed with $0.4 \mathrm{~mol} / 1$ sodium hydroxide solution $(250 \mu \mathrm{l})$ and left to stand at room temperature for $5 \mathrm{~min}$. LDH absorbance was measured at $450 \mathrm{~nm}$, ALT and AST absorbance values were measured at $510 \mathrm{~nm}$, and ALP absorbance was measured at $405 \mathrm{~nm}$ using a Multiskan ${ }^{\mathrm{TM}} \mathrm{GO}$ microplate reader (Thermo Fisher Scientific, Inc.).

$R T-q P C R$. Total RNA of cells were extracted using TRIzol ${ }^{\circledR}$ reagent (cat. no. 15596018; Thermo Fisher Scientific, Inc.), and RNA concentration was measured using a UV1700PC Nanodrop spectrophotometer (Nanodrop Technologies; Thermo Fisher Scientific,Inc.) and diluted to $500 \mathrm{ng} / \mu \mathrm{l}$. Reverse transcription was performed using Superscript II first-strand cDNA synthesis System (Invitrogen; Thermo Fisher Scientific, Inc.). The mRNA expression levels were determined by RT-qPCR using SYBR Green Real Time PCR kit (cat. no. 204057; Qiagen China, Co., Ltd.) in a 7500 Real-Time PCR system (Applied Biosystems, Inc.). A volume of $0.5 \mu \mathrm{l}$ forward primer $(10 \mu \mathrm{M}), 0.5 \mu \mathrm{l}$ reverse primer $(10 \mu \mathrm{M}), 4 \mu \mathrm{l} \mathrm{cDNA}$, $5 \mu \mathrm{l}$ SYBR Green I nucleic acid gel stain (cat. no. S9430; Sigma-Aldrich; Merck KGaA) were mixed into $10 \mu \mathrm{l}$ solution. The thermocycler conditions for the qPCR assay was set as follows: Pretreatment at $95^{\circ} \mathrm{C}$ for $1 \mathrm{~min}$, followed by 40 cycles of $95^{\circ} \mathrm{C}$ for $30 \mathrm{sec}, 58^{\circ} \mathrm{C}$ for $20 \mathrm{sec}$ and $70^{\circ} \mathrm{C}$ for $20 \mathrm{sec}$. The final extension was performed at $72^{\circ} \mathrm{C}$ for $7 \mathrm{~min}$ and then held at $4^{\circ} \mathrm{C}$. The sequences of primers are listed in Table I. The $2^{-\Delta \Delta \mathrm{Cq}}$ method was used to determine the expression levels of RT-PCR products (35).

Transfection. For the transfection assay, miR-148a-3p mimic (5'-UCAGUGCACUACAGAACUUUG-3'), ERBB3 overexpression plasmid (ERBB3-pcDNA3.1) and negative control (NC) were synthesized by Sangon Biotech Co., Ltd. The cells were digested, thoroughly mixed and seeded at $1 \times 10^{6} / \mathrm{ml}$ into 
the 6-well plate. The next day, the cells were transfected after reaching 80-90\% confluence. Briefly, $20 \mathrm{pmol}$ mimic or $1 \mu \mathrm{g}$ plasmid DNA was dissolved into $50 \mu 1$ Dulbecco's modified Eagle medium (DMEM; Hyclone; GE Healthcare) and mixed together with the transfection solution A, respectively. A total of $50 \mu \mathrm{l}$ DMEM was dissolved in $1 \mu \mathrm{l}$ Lipofectamine ${ }^{\circledR} 2000$ (Invitrogen; Thermo Fisher Scientific, Inc.), set aside for $5 \mathrm{~min}$ at room temperature and then mixed with the transfection solution A as the transfection solution B. Finally, the transfection solution B was added into the corresponding wells of the 6-well plate, and the cell culture plate was placed in a cell culture box at $37^{\circ} \mathrm{C}$ with $5 \% \mathrm{CO}_{2}$ for further culture. The culture medium was refreshed $24 \mathrm{~h}$ after the transfection, and then the cells were collected after $72 \mathrm{~h}$.

Luciferase activity assay. For dual-luciferase reporter assays, sequence information of ERBB 3' untranslated region (UTR) region was extracted and constructed into the pMIR-report Luciferase vector (Promega Corporation) to construct ERBB3 wild-type (WT) vector (3' UTR-WT). The target-binding region of miR-148a-3p and ERBB3 was predicted using TargetScan version 7.2 (http://www.targetscan.org/vert_72/). As there were 2 binding sites identified between miR-148a-3p and ERBB3, the 2 sites were mutated simultaneously to construct an ERBB3 mutant (MUT) plasmid (3' UTR-MUT). The pmirGLO vectors containing WT and MUT ERBB3 3' UTR were co-transfected with miR-148a-3p mimic into HSC-T6 cells using Lipofectamine ${ }^{\circledR} 2000$ (Invitrogen; Thermo Fisher Scientific, Inc.). A total of $48 \mathrm{~h}$ after transfection, the relative luciferase activities of the cells were measured by Dual-Luciferase Reporter Assay (Promega Corporation). Renilla luciferase activity was used for normalization.

Cell viability detection. The cells were inoculated into the 96-well plate at $1 \times 10^{5}$ per well, and cultured with $10 \%$ fetal bovine serum (FBS; Gibco; Thermo Fisher Scientific, Inc.) at $37^{\circ} \mathrm{C}$ and $5 \% \mathrm{CO}_{2}$. Next, $20 \mu 1 \mathrm{MTT}$ solution (cat. no. C0009; Beyotime Institute of Biotechnology) was added into each well and incubated for $4 \mathrm{~h}$. Then, $150 \mu \mathrm{l} 0.5 \%$ DMSO were added and gently shaken for $10 \mathrm{~min}$ to fully dissolve the purple crystals. Absorbance at $492 \mathrm{~nm}$ was read using a microplate reader (cat. no. E0225; Beyotime Institute of Biotechnology). All experiments were repeated 3 times and the average value was calculated.

Cell apoptosis. Following transfection for $24 \mathrm{~h}$, the cells $\left(1 \times 10^{6} / \mathrm{ml}\right)$ were resuspended in the solution composed of $500 \mu 1$ 1X Annexin binding buffer (V13246; Thermo Fisher Scientific, Inc.), $5 \mu \mathrm{l}$ fluorescein isothiocyanate (FITC) Annexin V (C1062S; Beyotime Institute of Biotechnology) and $1 \mu \mathrm{l} 100 \mu \mathrm{g} / \mathrm{ml}$ propidium iodide (P1304MP Thermo Fisher Scientific, Inc.). Then, after the reaction, $300 \mu 1 \mathrm{1X}$ Annexin Binding Buffer was added to the cell suspension for $15 \mathrm{~min}$ at room temperature. Finally, the stained cells were analyzed using a FACSCalibur ${ }^{\mathrm{TM}}$ flow cytometer (BD Bioscience) and FlowJo software (v.10.0 (FlowJo LLC).

Western blotting. The total proteins of cells were lysed using RIPA buffer (Tianjin Yitailong Science \& Technology Co.,
Ltd.). Next, the proteins (30 $\mu \mathrm{g} / \mathrm{lane})$ were boiled for $5 \mathrm{~min}$ at $100^{\circ} \mathrm{C}$ for denaturation, separated using $15 \%$ SDS-PAGE and then transferred to polyvinylidene fluoride membranes (EMD Millipore). The membranes were blocked using 5\% milk at room temperature for $1 \mathrm{~h}$. The blots were then probed with the primary antibodies: Rabbit anti-alpha-smooth muscle actin (anti- $\alpha$-SMA; 1:1,000; cat. no. ab32575; Abcam); rabbit anti-collagen I (1:1,000; cat. no. ab34710; Abcam); rabbit anti-Bcl-2 (1:1,000; cat. no. ab59348; Abcam); rabbit anti-Bax (1:1,000; cat. no. ab32503; Abcam); rabbit anti-ERBB3 (1:1,000; cat. no. ab5470; Abcam); rabbit anti-cleaved Caspase-3 (1:1,000; cat. no. ab2302; Abcam); and rabbit anti-GAPDH $\left(1: 2,000\right.$; cat. no. ab181602; Abcam) at $4^{\circ} \mathrm{C}$ overnight. The membranes were washed using PBS 3 times, and then incubated with goat anti-rabbit IgG $(\mathrm{H}+\mathrm{L})$ horseradish peroxidase-conjugated secondary antibody $(1: 2,000$; cat. no. SA00001-2; ProteinTech Group, Inc.) for $2 \mathrm{~h}$ at room temperature. ECL (Thermo Scientific, Thermo Fisher Scientific, Inc.) was used to detect the protein bands, which were then scanned by a multifunctional imager with Image $\mathbf{J}$ software v.4.7 (National Institutes of Health).

Statistical analysis. GraphPad Prism 6 (v.6.01; GraphPad Software, Inc.) was used for data analysis. The data were shown as mean \pm standard deviation of at least 3 independent experiments, and the differences between continuous variables from two groups was compared by t-test. A one-way analysis of variance (ANOVA) followed by Tukey's post hoc test was performed to analyze the differences among multiple groups. $\mathrm{P}<0.05$ was considered to indicate a statistically significant difference.

\section{Results}

miR-148a-3p expression in ALF model in rats and the degree of liver injury of model. The expression of miR-148a-3p decreased significantly in the ALF model of rats; however, miR-148a-3p expression in the group treated by miR-148a-3p was significantly increased compared with that of the model group ( $\mathrm{P}<0.001$; Fig. 1A). The expression levels of AST, ALT, ALP and LDH were markedly upregulated in the model, but significantly downregulated by miR-148a-3p $(\mathrm{P}<0.001$; Fig. 1B-E). Moreover, the liver indices increased significantly when the rats were treated with alcohol, but the indices decreased noticeably following miR-148a-3p treatment $(\mathrm{P}<0.001$; Fig. 1F).

Pathological changes of ALF model of rats. As shown in Fig. 2, the H\&E staining results after 9 weeks indicated that the hepatocytes in the liver tissues of rats in the normal control group were normal in size, clear in structure and abundant in cytoplasm. However, in the ALF model group, a large amount of steatosis in the liver, massive inflammatory cell infiltration, obvious interlobular connective tissue hyperplasia and formation of fibrous septum were observed. Moreover, treatment with miR-148a-3p mimics partially inhibited the liver fibrosis-like changes in rats in the alcohol model group. Compared with the model group, the model group treated with miR-148a-3p exhibited less infiltration of inflammatory cells in the liver and less fatty degeneration (Fig. 2). 

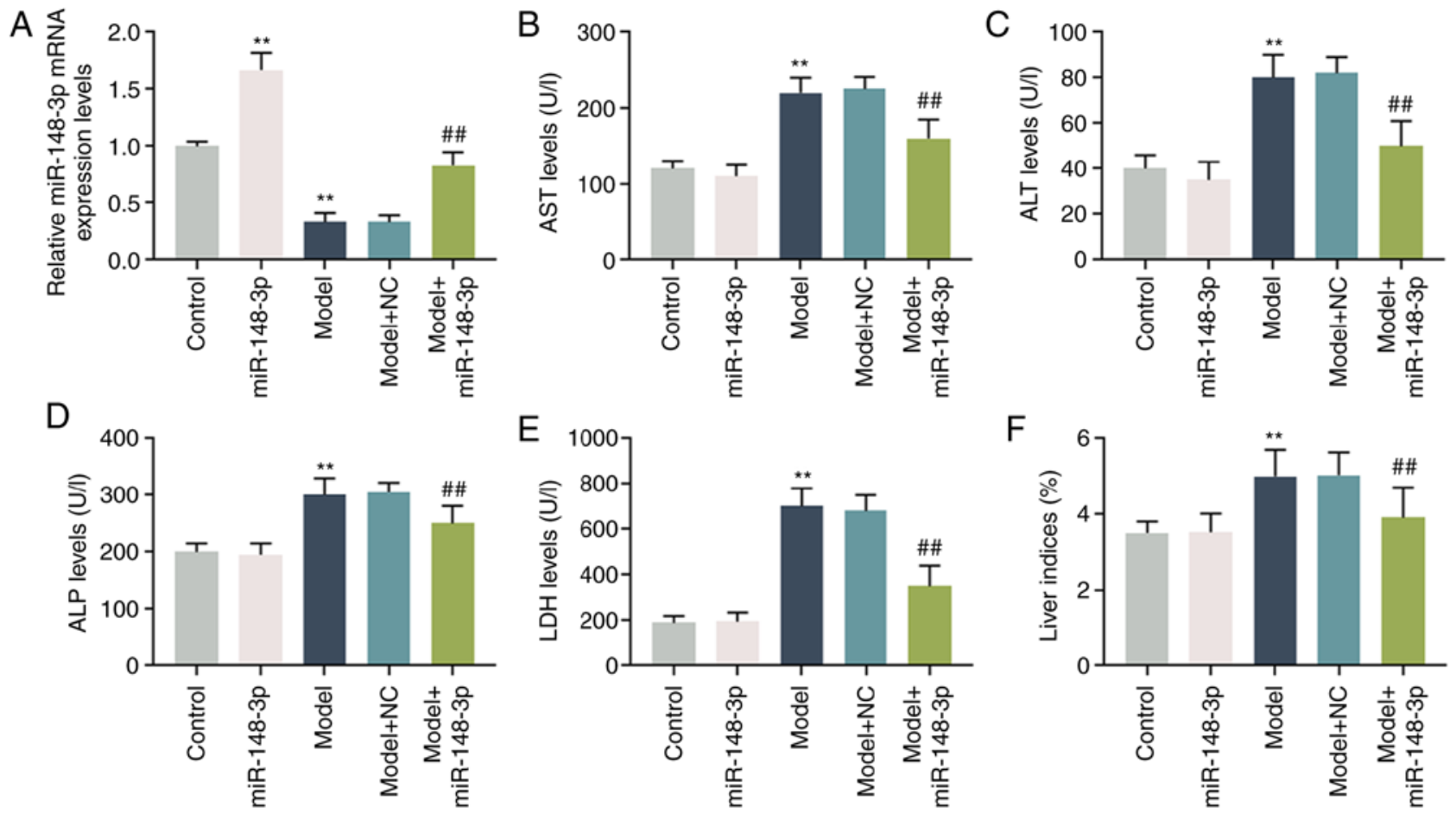

Figure 1. miR-148a-3p expression in rat alcoholic liver fibrosis model and the degree of liver injury. (A) Reverse transcription-quantitative PCR was performed to detect the expression of miR-148a-3p in liver tissues. The levels of (B) AST, (C) ALT, (D) ALP and (E) LDH were determined by enzyme-labeled colorimetry. (F) The ratio of the wet weight of the liver tissues to the body weight of the rats was termed the liver index. $(\mathrm{n}=3)^{* *} \mathrm{P}<0.001 \mathrm{vs}$. control. ${ }^{\# \#} \mathrm{P}<0.001 \mathrm{vs}$. model. miR, microRNA; AST, aspartate aminotransferase; ALT, alanine transaminase; ALP, alkaline phosphatase; LDH, lactic dehydrogenase; NC, negative control.
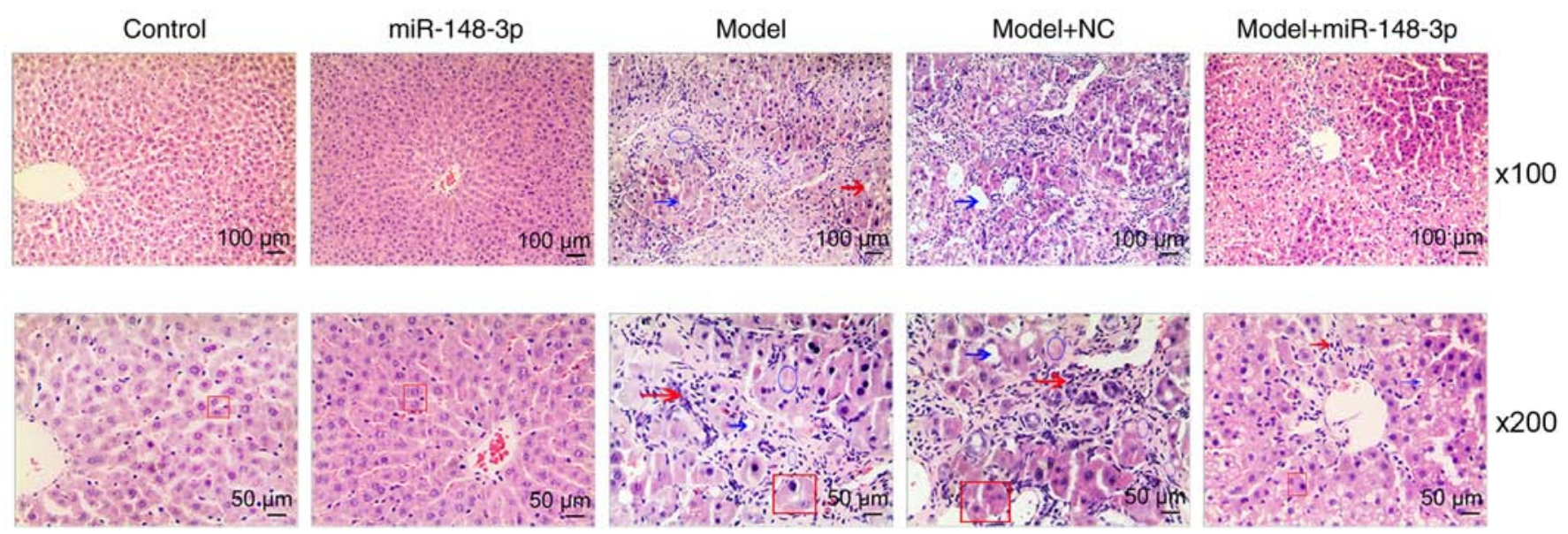

Figure 2. Pathological changes of alcoholic liver fibrosis model in rat liver tissues. Hematoxylin and eosin staining was used to observe the effect of miR-148a-3p on the degree of liver damage following alcohol gavage. The red arrows indicate inflammatory cell infiltration, the blue arrows highlight circular spaces within the tissue, indicative of steatosis, the red rectangles enclose the range of two healthy hepatocytes and the blue circles indicate fibrosis. miR, microRNA; NC, negative control.

Fibrosis in the ALF model. RT-qPCR and western blotting were performed to detect the expression levels of $\alpha$-SMA and type I collagen, and the results indicated that the expression levels of $\alpha$-SMA and type I collagen in the model group were significantly increased compared with those in the normal control group. Compared with the model group, following miR-148a-3p intervention, the protein expression levels of $\alpha$-SMA and type I collagen in the liver tissues were significantly downregulated, but still increased compared with those in the normal control group (P<0.001; Fig. 3A-C).
ERBB3 directly targets miR-148a-3p in liver cells. TargetScan7.2 software (http://www.targetscan.org/vert_72/) predicted that miR-148a-3p and ERBB3 shared binding sites (Fig. 4A). PmirGLO dual-luciferase reporter vectors were constructed by respectively transfecting ERBB3-WT and ERBB3-MUT with miR-148a-3p mimic into HSC-T6 cells, and it was identified that the levels of luciferase activity in the ERBB3-WT cells were significantly suppressed $(\mathrm{P}<0.001$; Fig. 4B). In addition, the association between ERBB3 and miR-148a-3p was explored. Firstly, the miR-148a-3p mimic was transfected into HSC-T6 cells and the transfection 

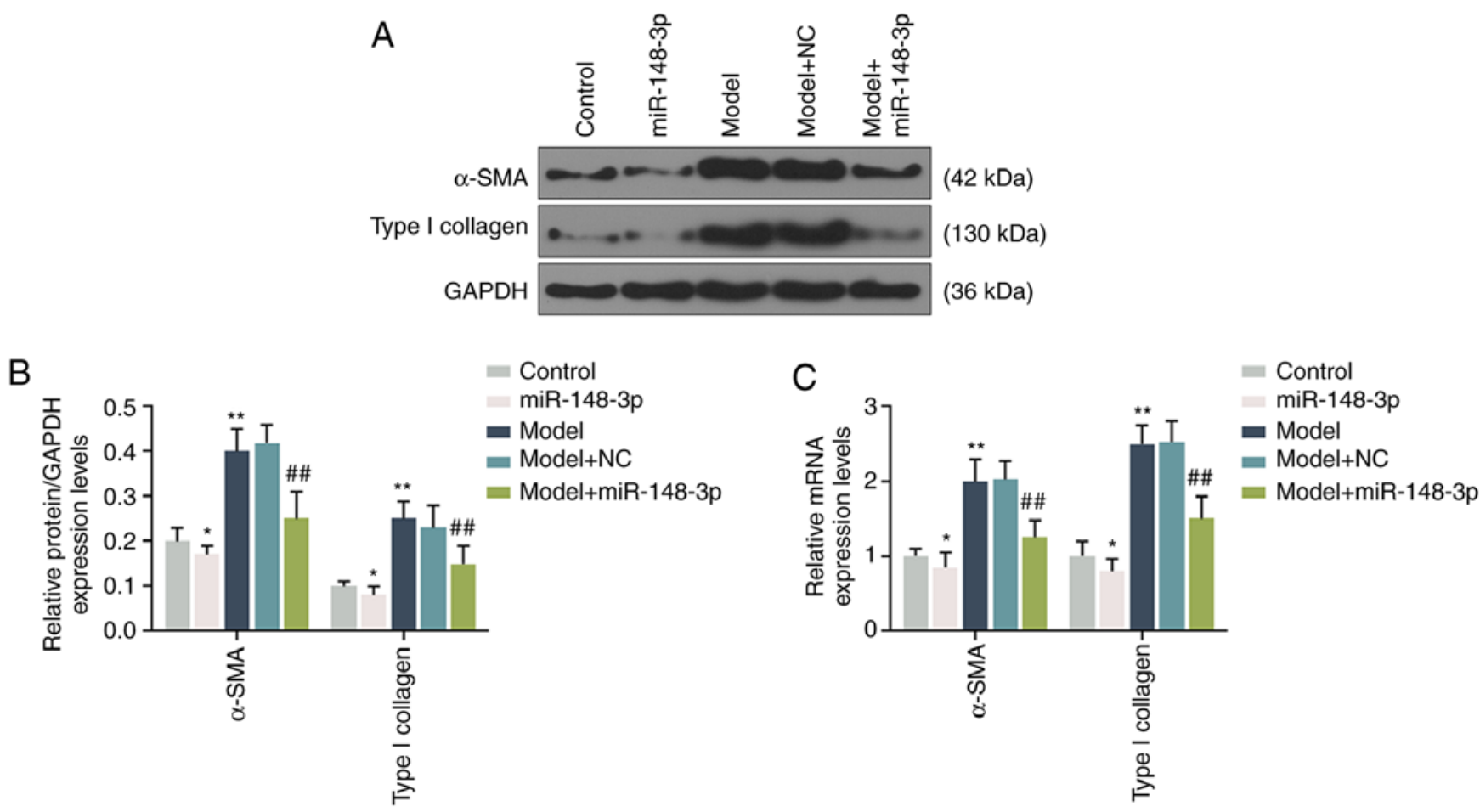

Figure 3. Expression levels of $\alpha$-SMA and type I collagen in the liver tissue. (A and B) Western blotting and (C) reverse transcription-quantitative PCR were used to detect the effects of miR-148a-3p on the protein and mRNA expression levels of $\alpha$-SMA and type I collagen in liver tissues following alcohol gavage. $(\mathrm{n}=3) .{ }^{*} \mathrm{P}<0.05$ and $^{* *} \mathrm{P}<0.001$ vs. control. ${ }^{\# \#} \mathrm{P}<0.001$ vs. Model + NC. miR, microRNA; NC, negative control; $\alpha$-SMA, alpha-smooth muscle actin.
A Position 679-686 of ERBB3 3' UTR 5' ... UARAGCUGUUCUUUA-UGCACUGA... 3, IIIII IIIIIII $8 \mathrm{mer}$

B

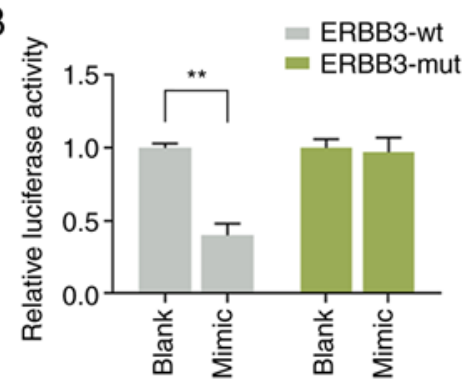

E

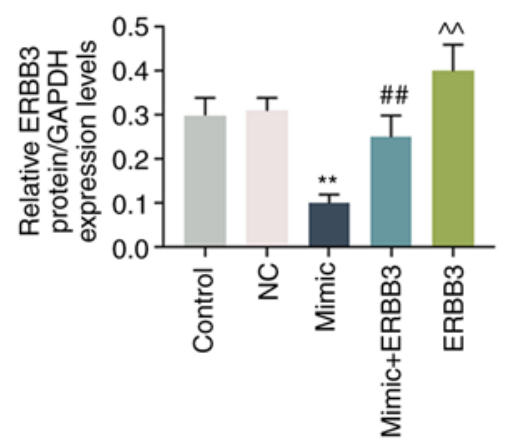

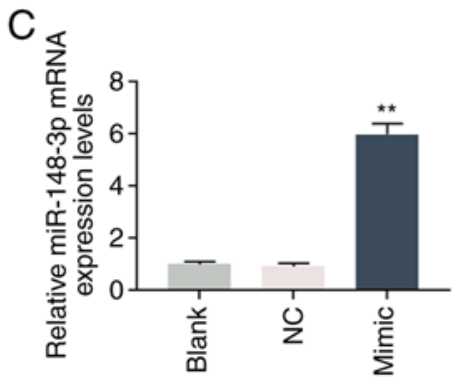

D

$\mathrm{F}$

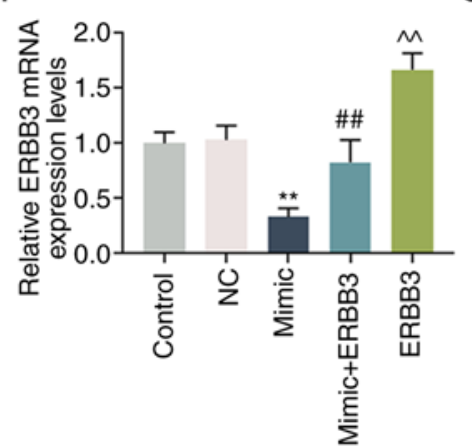

$\mathrm{G} \odot$

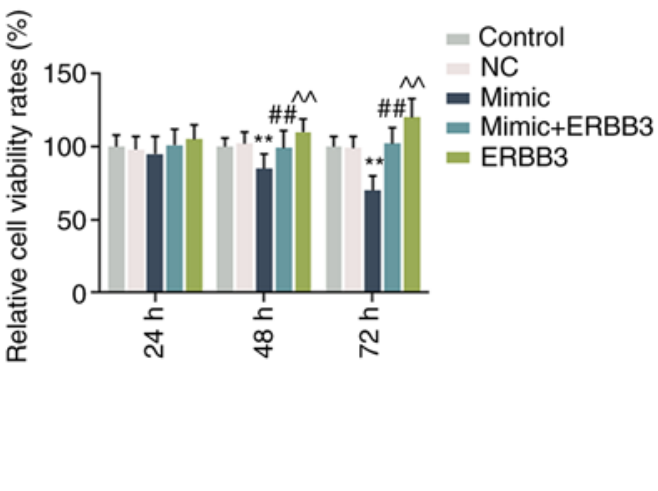

Figure 4. ERBB3 directly targeted miR-148a-3p in HSC-T6 cells. (A) TargetScan7.2 was used to predict the potential target gene of miR-148a-3p. (B) A dual-luciferase reporter assay was used to analyze the fluorescence activity of ERBB3 in miR-148a-3p-overexpressing cells $(n=3){ }^{* * *} \mathrm{P}<0.001 \mathrm{vs}$. Blank. (C) RT-qPCR analysis showing the transfection efficiency of miR-148a-3p mimic in HSC-T6 cells. ${ }^{* *} \mathrm{P}<0.001$ vs. Blank. (D and E) Western blotting was used to determine the expression of ERBB3 in cells overexpressing miR-148a-3p. (F) RT-qPCR was used to analyze the expression of ERBB3 in cells overexpressing miR-148a-3p. $(\mathrm{n}=3) .{ }^{* *} \mathrm{P}<0.001$ vs. Control. ${ }^{\# \#} \mathrm{P}<0.001$ vs. Mimic. ${ }^{\wedge} \mathrm{P}<0.001$ vs. Mimic + ERBB3. $(\mathrm{G})$ Cell viability was measured by MTT assay $(\mathrm{n}=3)$. ${ }^{* *} \mathrm{P}<0.001$ vs. control. ${ }^{\# \#} \mathrm{P}<0.001$ vs. mimic. ${ }^{\wedge} \mathrm{P}<0.001$ vs. Mimic + ERBB3. ERBB3, receptor tyrosine-protein kinase erbB-3; miR, microRNA; RT-qPCR, reverse transcription-quantitative PCR; UTR, untranslated region; wt, wild-type; mut, mutant; NC, negative control. 

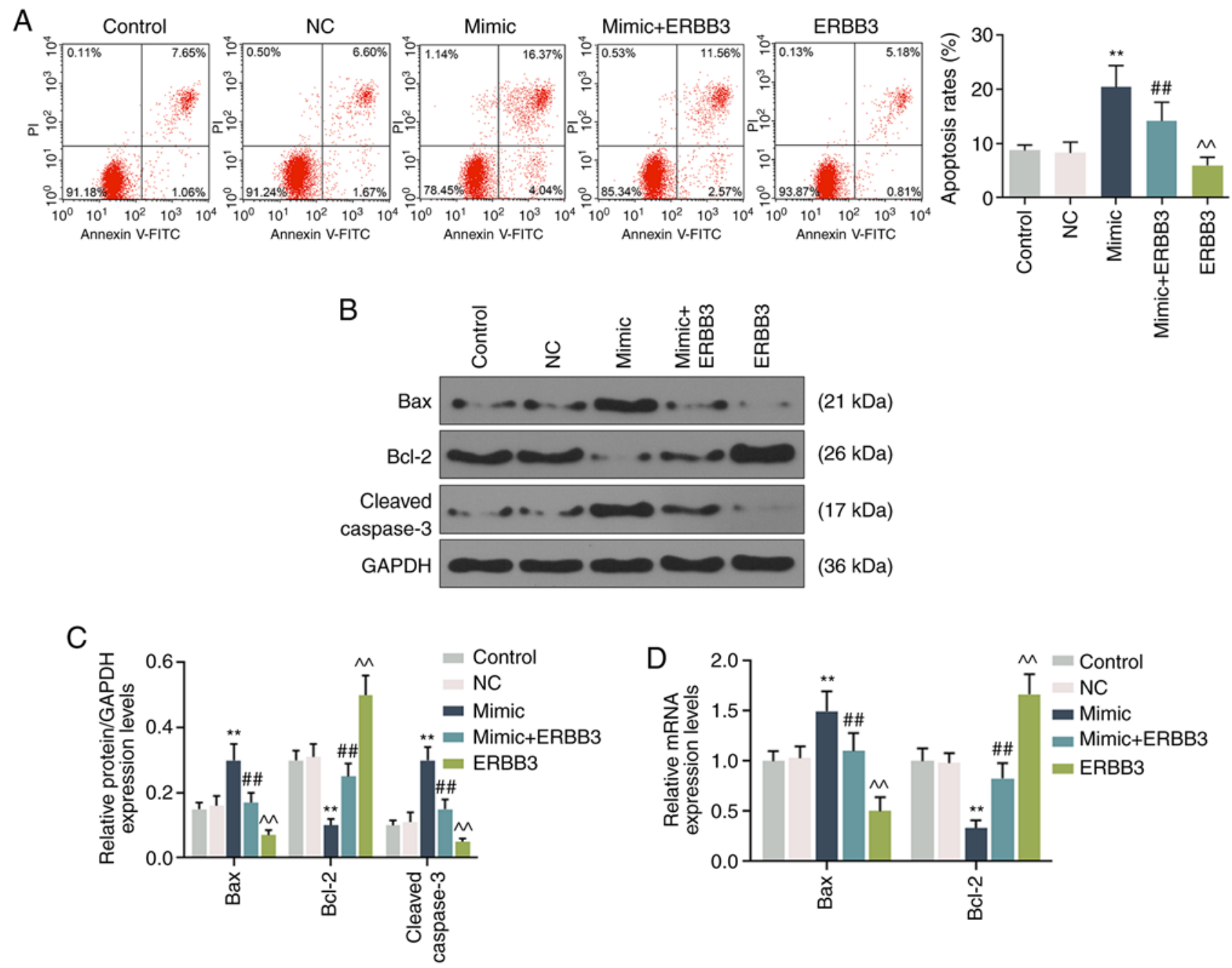

Figure 5. Effects of miR-148a-3p on the viability and apoptosis of HSC-T6 cells by targeting ERBB3. (A) Cell apoptosis was detected by flow cytometry. (B and C) Western blotting was used to determine the protein expression levels of Bax, Bcl-2 and cleaved caspase-3 in cells. (D) The mRNA expression levels of Bax, Bcl-2 and cleaved caspase- 3 in cells were detected by reverse transcription-quantitative PCR. $(\mathrm{n}=3){ }^{*}{ }^{* *} \mathrm{P}<0.001$ vs. Control. ${ }^{\# \# / 4} \mathrm{P}<0.001$ vs. mimic. ${ }^{\wedge} \mathrm{P}<0.001$ vs. mimic + ERBB3. miR, microRNA; ERBB3, receptor tyrosine-protein kinase erbB-3; NC, negative control.

efficiency was assessed using RT-qPCR (Fig. 4C). Next, cells were transfected with miR-148a-3p mimics, ERBB3 overexpression plasmids or co-transfected with miR-148a-3p mimics and ERBB3 overexpression plasmids. It was identified that the expression of ERBB3 decreased noticeably in HSC-T6 cells transfected with miR-148a-3p mimic, but was significantly increased when the mimic + ERBB3 was transfected into the cells $(\mathrm{P}<0.001$; Fig. 4D-F).

Effects of miR-148a-3p on the viability and apoptosis of liver cells by targeting ERBB3. The cells were transfected with miR-148a-3p mimic and ERBB3 overexpression plasmids for $48 \mathrm{~h}$. The data demonstrated that the cell viability was decreased significantly in cells transfected with the mimic, but markedly increased following transfection with the ERBB3 plasmid. However, the mimic inhibited the effect of the ERBB3 plasmids on the promotion of cell viability ( $\mathrm{P}<0.001$; Fig. 4G). Similarly, transfection with the mimic reversed the effect of ERBB3 on inhibiting cell apoptosis $(\mathrm{P}<0.001$; Fig. 5A). Concomitantly, the Bcl-2 expression level was observed to be significantly decreased, but the expression levels of Bax and c-cleaved-3 were markedly increased in the cells treated by
miR-148a-3p. Moreover, following ERBB3 transfection, the expression level of these apoptosis-associated proteins showed the opposite results, however, mimic transfection reversed the effect of ERBB3 on the expression levels of these proteins $(\mathrm{P}<0.001$; Fig. 5B-D).

\section{Discussion}

Since the majority of miRNAs involved in various physiological and pathological processes are highly conserved, and miRNA expression is tissue-specific, studying the characteristics of miRNAs can provide novel insights into the detection and treatment of certain associated diseases $(36,37)$. Alcoholic liver disease, which is a common condition affecting the digestive system, is characterized by impaired liver function (38). In previous studies, miRNAs have been identified to serve an important role in cell differentiation and function of the liver; therefore, the comprehensively study of miRNAs may also provide novel insights into the pathogenesis of alcoholic liver disease in humans $(39,40)$. It has been demonstrated that genes from the miR-148 family, for example, miR-148a (miR-148a-3p), serve important roles in physiological liver function through 
promoting the hepatospecific phenotype and inhibiting the invasiveness of transformed cells $(18,19)$. miR-148a-3p is also considered to act as a tumor suppressor gene of hepatocellular carcinoma, and has potential value in evaluating the prognosis of patients with hepatocellular carcinoma (41). In addition, miR-148a knockout promoted liver lipid metabolism in mice (42). However, the role of the miR-148 family in the regulation of alcoholic liver disease has not been reported in detail, and therefore became the focus of the present study.

Previous data revealed that the expression levels of miR-132 and miR-155 in the liver and brain were significantly increased following long-term consumption of alcohol in mice (43). However, a study conducted on the association between liver aging and miRNA expression profiles in rats identified that miR-148a-3p expression was greatly downregulated in the livers of aged rats (44). Therefore, the present study further explored the association between miR-148a-3p and alcoholic liver disease in rats. The rats were treated with miR-148-3p and received alcohol gavage for 9 weeks. The results demonstrated that the expression of miR-148a-3p in the livers of the alcohol-treated rats was significantly decreased; moreover, the miR-148a-3p expression levels in rats treated with miR-148a-3p mimics were promoted. These results were consistent with those from the study by Mimura et al (44).

In previous years, the mechanisms of the involvement of miRNAs in the pathological process of liver diseases have been widely studied. For example, Bala et al (14) identified that miR-155 has a certain protective effect on alcohol-treated mouse liver, and may decreased oxidative stress and steatosis. miR-122 was also observed to protect liver function by lowering AST and ALT levels, which are used as markers of liver injury (45). Therefore, the present study investigated the effect of miR-148a-3p on the liver pathology of the rats with alcoholic liver disease. LDH, ALT, AST and ALP are used as indicators to evaluate the degree of liver injury, as they have high levels in various liver diseases (46-48). The present study identified that miR-148a-3p inhibited the expression levels of AST, ALT, ALP and LDH in the model. In addition, a higher liver index indicates a larger amount of lipid deposition in the liver. In the present study, following treatment with miR-148a-3p mimics in the rats, it was identified that the liver indices of the rats treated with ethanol were significantly decreased compared with the blank control group, indicating the protective effect of miR-148a-3p in the rat model.

Overconsumption of alcohol can increase levels of hepatic fibrosis, which involves the increase of activated fibroblasts or myofibroblasts when necrosis occurs in hepatocytes. It is a tissue repair process observed in various chronic liver injuries, and can result in cirrhosis in severe cases $(49,50)$. Increased $\alpha$-SMA and type I collagen expression levels are important markers of liver fibrosis (51), and according to the results of the present study, miR-148a-3p treatment markedly inhibited $\alpha$-SMA and type I collagen expression levels in the ALF rats, indicating that miR-148a-3p can improve liver fibrosis caused by alcohol.

A previous study showed that mir-148a-3p can bind to other target genes to regulate the progress of various diseases, and miR-148a can inhibit the proliferation and migration of pancreatic cancer cells by downregulating the expression of ERBB3 (52). miR-148a-3p in combination with ERBB3 inhibited the proliferation of bladder cancer and epithelial mesenchymal transition (21). In addition, ERBB3 overexpression can lead to cell degeneration and the occurrence and development of tumors, and is currently considered as a proto-oncogene with important research and clinical application potential $(53,54)$. In the present study, TargetScan7.2 software predicted that ERBB3 was a possible target gene of miR-148a-3p, which was further confirmed by a luciferase reporter assay. Furthermore, ERBB3 overexpression plasmids were transfected with miR-148a-3p mimic into hepatic stellate HSC-T6 cells, and the experimental results confirmed that the overexpression of miR-148a-3p could inhibit the expression of ERBB3 in the hepatic stellate cells.

The level of ERBB3 is closely associated with the risk of developing liver cancer $(55,56)$. The absence of ERBB3 in hepatocytes affects the occurrence of liver cancer and can delay the formation of liver tumors and cell proliferation (29). Besides that, it has been reported that EGFR-ERBB3 signaling in hepatocytes is also required for the activation of hepatic stellate cells and liver fibrosis (27). The present study identified that ERBB3 can promote cell viability, inhibit cell apoptosis of stellate cells, and that the expression of miR-148a-3p partially reversed the effects of ERBB3 on promoting cell viability and suppressing cell apoptosis, suggesting that miR-148a-3p can be targeted by ERBB3 to regulate the activation of hepatic stellate cells.

The present study identified a significant effect of miR-148-a-3p on rat liver fibrosis. It was also observed that ERBB3 may be the downstream gene of miR-148-a-3p in hepatic stellate cells. Nevertheless, the present study had a number of limitations, due to funding limitations. Firstly, the effect of miR-148-a-3p/ERBB3 signaling axis on hepatic stellate cell fibrosis was not detected. Secondly, whether the miR-148-a-3p/ERBB3 signaling axis and associated function identified in HSC-T6 cells are consistent with those in rats primary hepatic stellate cells requires further exploration. Besides, whether the miR-148-a-3p/ERBB3 signaling axis and its associated function contribute to the liver fibrosis in vivo also requires confirmation.

In conclusion, the present study revealed that miR-148-a-3p regulated ALF, and the viability and apoptosis of hepatic stellate cells through targeting ERBB3. However, since in vivo experiments were only performed in rats in the present study, further human sample collection is required to verify the results.

\section{Acknowledgements}

Not applicable.

\section{Funding}

The present study was supported by the National Natural Science Foundation of China (grant nos. 81700509 and 81400663) and the Fundamental Research Funds for the Central Universities (grant no. 22120180619).

\section{Availability of data and materials}

The analyzed data sets generated during the study are available from the corresponding author on reasonable request. 


\section{Authors' contributions}

JX was responsible for substantial contributions to the conception and design of the study, and agreed to be accountable for all aspects of the work in ensuring that questions related to the accuracy or integrity of the work are appropriately investigated and resolved. JN was involved in data acquisition, data analysis and interpretation. $\mathrm{CC}$ and $\mathrm{KW}$ were involved in drafting the article or critically revising it for important intellectual content. All authors provided final approval of the version to be published.

\section{Ethics approval and consent to participate}

The animal experimental protocol was approved by the Tongji Hospital of Tongji University and performed in accordance with the Guidance and Ethics Committee of Tongji Hospital, Tongji University School of Medicine (approval no. THTU20160810A).

\section{Patient consent for publication}

Not applicable.

\section{Competing interests}

The authors declare that they have no competing interests.

\section{References}

1. Ding L, Wo L, Du Z, Tang L, Song Z and Dou X: Danshen protects against early-stage alcoholic liver disease in mice via inducing PPAR $\alpha$ activation and subsequent 4-HNE degradation. PLoS One 12: e0186357, 2017.

2. Singal AK, Kamath PS, Gores GJ and Shah VH: Alcoholic hepatitis: Current challenges and future directions. Clin Gastroenterol Hepatol 12: 555-564; quiz e31-e32, 2014.

3. Gao B and Bataller R: Alcoholic liver disease: Pathogenesis and new therapeutic targets. Gastroenterology 141: 1572-1585, 2011.

4. Bataller R and Gao B: Liver fibrosis in alcoholic liver disease. Semin Liver Dis 35: 146-156, 2015.

5. Voican CS, Louvet A, Trabut JB, Njiké-Nakseu M, Dharancy S, Sanchez A, Corouge M, Lamouri K, Lebrun A, Balian A, et al: Transient elastography alone and in combination with FibroTest ${ }^{\circledR}$ for the diagnosis of hepatic fibrosis in alcoholic liver disease. Liver Int 37: 1697-1705, 2017.

6. Roh YS and Seki E: Toll-like receptors in alcoholic liver disease, non-alcoholic steatohepatitis and carcinogenesis. J Gastroenterol Hepatol 28 (Suppl 1): S38-S42, 2013.

7. Devi BG, Henderson GI, Frosto TA and Schenker S: Effect of ethanol on rat fetal hepatocytes: Studies on cell replication, lipid peroxidation and glutathione. Hepatology 18: 648-659, 1993.

8. Olguín-Martínez M, Hernández-Espinosa DR and HernándezMuñoz R: High $\alpha$-tocopherol dosing increases lipid metabolism by changing redox state in damaged rat gastric mucosa and liver after ethanol treatment. Clin Sci (Lond) 132: 1257-1272, 2018.

9. Li YL, Wu J, Wei D, Zhang DW, Feng H, Chen ZN and Bian H: Newcastle disease virus represses the activation of human hepatic stellate cells and reverses the development of hepatic fibrosis in mice. Liver Int 29: 593-602, 2009.

10. Higashi T, Friedman SL and Hoshida Y: Hepatic stellate cells as key target in liver fibrosis. Adv Drug Deliv Rev 121: 27-42, 2017.

11. Yu HX, Yao Y, Bu FT, Chen Y, Wu YT, Yang Y, Chen X, Zhu Y, Wang Q, Pan XY, et al: Blockade of YAP alleviates hepatic fibrosis through accelerating apoptosis and reversion of activated hepatic stellate cells. Mol Immunol 107: 29-40, 2019.

12. Andueza A, Garde N, García-Garzón A, Ansorena E, López-Zabalza MJ, Iraburu MJ, Zalba G and Martínez-Irujo JJ: NADPH oxidase 5 promotes proliferation and fibrosis in human hepatic stellate cells. Free Radic Biol Med 126: 15-26, 2018.
13. Hartmann P and Tacke F: Tiny RNA with great effects: miR-155 in alcoholic liver disease. J Hepatol 64: 1214-1216, 2016.

14. Bala S, Csak T, Saha B, Zatsiorsky J, Kodys K, Catalano D, Satishchandran A and Szabo G: The pro-inflammatory effects of miR-155 promote liver fibrosis and alcohol-induced steatohepatitis. J Hepatol 64: 1378-1387, 2016.

15. Leti F, Malenica I, Doshi M, Courtright A, Van Keuren-Jensen K, Legendre C, Still CD, Gerhard GS and DiStefano JK: High-throughput sequencing reveals altered expression of hepatic microRNAs in nonalcoholic fatty liver disease-related fibrosis. Transl Res 166: 304-314, 2015.

16. Hayes J, Peruzzi PP and Lawler S: MicroRNAs in cancer: Biomarkers, functions and therapy. Trends Mol Med 20: 460-469, 2014.

17. Trionfini $P$ and Benigni A: MicroRNAs as master regulators of glomerular function in health and disease. J Am Soc Nephrol 28: 1686-1696, 2017.

18. Gailhouste L, Gomez-Santos L, Hagiwara K, Hatada I, Kitagawa N, Kawaharada K, Thirion M, Kosaka N, Takahashi RU, Shibata T, et al: miR-148a plays a pivotal role in the liver by promoting the hepatospecific phenotype and suppressing the invasiveness of transformed cells. Hepatology 58: 1153-1165, 2013.

19. Jung KH,Zhang J,Zhou C,Shen H,Gagea M,Rodriguez-AguayoC, Lopez-Berestein G, Sood AK and Beretta L: Differentiation therapy for hepatocellular carcinoma: Multifaceted effects of miR-148a on tumor growth and phenotype and liver fibrosis. Hepatology 63: 864-879, 2016.

20. Bhattacharya S, Chalk AM, Ng AJ, Martin TJ, Zannettino AC, Purton LE, Lu J, Baker EK and Walkley CR: Increased miR-155-5p and reduced miR-148a-3p contribute to the suppression of osteosarcoma cell death. Oncogene 35: 5282-5294, 2016.

21. Wang X, Liang Z, Xu X, Li J, Zhu Y, Meng S, Li S, Wang S, Xie B, Ji A, et al: miR-148a-3p represses proliferation and EMT by establishing regulatory circuits between ERBB3/AKT2/ c-myc and DNMT1 in bladder cancer. Cell Death Dis 7: e2503, 2016.

22. Bellissimo T, Russo E, Ganci F, Vico C, Sacconi A, Longo F, VitoloD, Anile M, DisioD, Marino M, et al: Circulating miR-21-5p and miR-148a-3p as emerging non-invasive biomarkers in thymic epithelial tumors. Cancer Biol Ther 17: 79-82, 2016.

23. Chen W, Zhao W, Yang A, Xu A, Wang H, Cong M, Liu T, Wang P and You H: Integrated analysis of microRNA and gene expression profiles reveals a functional regulatory module associated with liver fibrosis. Gene 636: 87-95, 2017.

24. Li Z, Wang J, Zeng Q, Hu C, Zhang J, Wang H, Yan J, Li H and Yu Z: Long noncoding RNA HOTTIP promotes mouse hepatic stellate cell activation via downregulating miR-148a. Cell Physiol Biochem 51: 2814-2828, 2018.

25. Zhu J, Luo Z, Pan Y, Zheng W, Li W, Zhang Z, Xiong P, Xu D, Du M, Wang B, et al: H19/miR-148a/USP4 axis facilitates liver fibrosis by enhancing TGF- $\beta$ signaling in both hepatic stellate cells and hepatocytes. J Cell Physiol 234: 9698-9710, 2019.

26. Liu XY, He YJ, Yang QH, Huang W, Liu ZH, Ye GR, Tang SH and Shu JC: Induction of autophagy and apoptosis by miR-148a through the sonic hedgehog signaling pathway in hepatic stellate cells. Am J Cancer Res 5: 2569-2589, 2015.

27. Scheving LA, Zhang X, Threadgill DW and Russell WE: Hepatocyte ERBB3 and EGFR are required for maximal CCl4-induced liver fibrosis. Am J Physiol Gastrointest Liver Physiol 311: G807-G816, 2016.

28. Cao K, Gong H, Qiu Z, Wen Q, Zhang B, Tang T, Zhou X, Cao T, Wang B, Shi $\mathrm{H}$ and Wang R: Hepatitis B virus X protein reduces the stability of Nrdp1 to up-regulate ErbB3 in hepatocellular carcinoma cells. Tumour Biol 37: 10375-10382, 2016.

29. Scheving LA, Zhang X, Stevenson MC, Weintraub MA, Abbasi A, Clarke AM, Threadgill DW and Russell WE: Loss of hepatocyte ERBB3 but not EGFR impairs hepatocarcinogenesis. Am J Physiol Gastrointest Liver Physiol 309: G942-G954, 2015.

30. Li G, Zhang W, Gong L and Huang X: MicroRNA 125a-5p inhibits cell proliferation and induces apoptosis in hepatitis $\mathrm{B}$ virus-related hepatocellular carcinoma by downregulation of ErbB3. Oncol Res 27: 449-458, 2019.

31. Shi J: Regulatory networks between neurotrophins and miRNAs in brain diseases and cancers. Acta Pharmacol Sin 36: 149-157, 2015.

32. Agarwal V, Bell GW, Nam JW and Bartel DP: Predicting effective microRNA target sites in mammalian mRNAs. Elife 4: $\mathrm{e} 05005,2015$. 
33. Ni YH, Huo LJ and Li TT: Antioxidant axis Nrf2-keap1-ARE in inhibition of alcoholic liver fibrosis by IL-22. World $\mathrm{J}$ Gastroenterol 23: 2002-2011, 2017.

34. Ma X, Luo Q, Zhu H, Liu X, Dong Z, Zhang K, Zou Y, Wu J, Ge J and Sun A: Aldehyde dehydrogenase 2 activation ameliorates $\mathrm{CCl}_{4}$-induced chronic liver fibrosis in mice by up-regulating Nrf2/HO-1 antioxidant pathway. J Cell Mol Med 22: 3965-3978, 2018.

35. Livak KJ and Schmittgen TD: Analysis of relative gene expression data using real-time quantitative PCR and the 2(-Delta Delta C(T)) method. Methods 25: 402-408, 2001.

36. Yuan DZ, Yu LL, Qu T, Zhang SM, Zhao YB, Pan JL, Xu Q, He YP, Zhang JH and Yue LM: Identification and characterization of progesterone- and estrogen-regulated MicroRNAs in mouse endometrial epithelial cells. Reprod Sci 22: 223-234, 2015.

37. Zhao F, Xu G, Zhou Y, Wang L, Xie J, Ren S, Liu S and Zhu Y: MicroRNA-26b inhibits hepatitis B virus transcription and replication by targeting the host factor CHORDC1 protein. J Biol Chem 289: 35029-35041, 2014.

38. Osaki K, Shimizu Y, Yamamoto T, Miyake F, Kondo S and Yamaguchi H: Improvement of liver function by the administration of oyster extract as a dietary supplement to habitual alcohol drinkers: A pilot study. Exp Ther Med 10: 705-710, 2015.

39. Zhang Y, Huang F, Wang J, Peng L and Luo H: MiR-15b mediates liver cancer cells proliferation through targeting BCL-2. Int J Clin Exp Pathol 8: 15677-15683, 2015.

40. Roderburg $C$ and Trautwein C: Cell-specific functions of miRNA in the liver. J Hepatol 66: 655-656, 2017.

41. Pan L, Huang S, He R, Rong M, Dang Y and Chen G: Decreased expression and clinical significance of miR-148a in hepatocellular carcinoma tissues. Eur J Med Res 19: 68, 2014

42. Cheng L, Zhu Y, Han H, Zhang Q, Cui K, Shen H, Zhang J, Yan J, Prochownik E and Li Y: MicroRNA-148a deficiency promotes hepatic lipid metabolism and hepatocarcinogenesis in mice. Cell Death Dis 8: e2916, 2017.

43. Bala S and Szabo G: MicroRNA signature in alcoholic liver disease. Int J Hepatol 2012: 498232, 2012.

44. Mimura S, Iwama $\mathrm{H}$, Kato $\mathrm{K}$, Nomura K, Kobayashi $\mathrm{M}$, Yoneyama H, Miyoshi H, Tani J, Morishita A, Himoto T, et al: Profile of microRNAs associated with aging in rat liver. Int J Mol Med 34: 1065-1072, 2014.

45. Roderburg C,BenzF, Vargas CardenasD,Koch A,JanssenJ, VucurM, Gautheron J, Schneider AT, Koppe C, Kreggenwinkel K, et al: Elevated miR-122 serum levels are an independent marker of liver injury in inflammatory diseases. Liver Int 35: 1172-1184, 2015.

46. Lv Y, Zhang B, Xing G, Wang F and Hu Z: Protective effect of naringenin against acetaminophen-induced acute liver injury in metallothionein (MT)-null mice. Food Funct 4: 297-302, 2013.
47. Rahim SM, Taha EM, Al-janabi MS, Al-douri BI, Simon KD and Mazlan AG: Hepatoprotective effect of cymbopogon citratus aqueous extract against hydrogen peroxide-induced liver injury in male rats. Afr J Tradit Complement Altern Med 11: 447-451, 2014.

48. Ferriero R, Nusco E, De Cegli R, Carissimo A, Manco G and Brunetti-Pierri N: Pyruvate dehydrogenase complex and lactate dehydrogenase are targets for therapy of acute liver failure. J Hepatol 69: 325-335, 2018.

49. Oh Y, Park O, Swierczewska M, Hamilton JP, Park JS, Kim TH, Lim SM, Eom H, Jo DG, Lee CE, et al: Systemic PEGylated TRAIL treatment ameliorates liver cirrhosis in rats by eliminating activated hepatic stellate cells. Hepatology 64: 209-223, 2016.

50. Yang JA, Kong WH, Sung DK, Kim H, Kim TH, Lee KC and Hahn SK: Hyaluronic acid-tumor necrosis factor-related apoptosis-inducing ligand conjugate for targeted treatment of liver fibrosis. Acta Biomater 12: 174-182, 2015.

51. Wang T, Wu D, Li P, Zhang K, Tao S, Li Z and Li J: Effects of Taohongsiwu decoction on the expression of $\alpha$-SMA and TGF- $\beta 1$ mRNA in the liver tissues of a rat model of hepatic cirrhosis. Exp Ther Med 14: 1074-1080, 2017.

52. Feng H, Wang Y, Su J, Liang H, Zhang CY, Chen X and Yao W: MicroRNA-148a suppresses the proliferation and migration of pancreatic cancer cells by down-regulating ErbB3. Pancreas 45: 1263-1271, 2016

53. Zhang K, Wong P, Salvaggio C, Salhi A, Osman I and Bedogni B: Synchronized targeting of Notch and ERBB signaling suppresses melanoma tumor growth through inhibition of Notch1 and ERBB3. J Invest Dermatol 136: 464-472, 2016.

54. Humtsoe JO, Pham E, Louie RJ, Chan DA and Kramer RH: ErbB3 upregulation by the HNSCC 3D microenvironment modulates cell survival and growth. Oncogene 35: 1554-1564, 2016.

55. Sayagués JM, Corchete LA, Gutiérrez ML, Sarasquete ME, Del Mar Abad M, Bengoechea O, Fermiñán E, Anduaga MF, Del Carmen S, Iglesias M, et al: Genomic characterization of liver metastases from colorectal cancer patients. Oncotarget 7: 72908-72922, 2016.

56. Shi DM, Li LX, Bian XY, Shi XJ, Lu LL, Zhou HX, Pan TJ, Zhou J, Fan J and Wu WZ: miR-296-5p suppresses EMT of hepatocellular carcinoma via attenuating NRG1/ERBB2/ERBB3 signaling. J Exp Clin Cancer Res 37: 294, 2018.

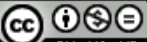

This work is licensed under a Creative Commons Attribution-NonCommercial-NoDerivatives 4.0 International (CC BY-NC-ND 4.0) License. 